\title{
Impact of static magnetic fields on human myoblast cell cultures
}

\author{
JENS STERN-STRAETER ${ }^{1 *}$, GABRIEL ALEJANDRO BONATERRA ${ }^{2 *}$, STEFAN S. KASSNER ${ }^{1}$, \\ ANNE FABER $^{1}$, ALEXANDER SAUTER ${ }^{1}$, JOHANNES D. SCHULZ ${ }^{1}$, KARL HÖRMANN ${ }^{1}$, \\ RALF KINSCHERF $^{2 * *}$ and ULRICH REINHART GOESSLER ${ }^{1 * *}$ \\ ${ }^{1}$ Department of Otolaryngology, Head and Neck Surgery, Medical Faculty Mannheim, \\ University of Heidelberg, D-68167 Mannheim; ${ }^{2}$ Department of Medical Cell Biology, \\ Anatomy and Cell Biology, University of Marburg, D-35032 Marburg, Germany
}

Received June 6, 2011; Accepted July 11, 2011

DOI: $10.3892 / \mathrm{ijmm} .2011 .777$

\begin{abstract}
Treatment of skeletal muscle loss due to trauma or tumor ablation therapy still lacks a suitable clinical approach. Creation of functional muscle tissue in vitro using the differentiation potential of human satellite cells (myoblasts) is a promising new research field called tissue engineering. Strong differentiation stimuli, which can induce formation of myofibers after cell expansion, have to be identified and evaluated in order to create sufficient amounts of neo-tissue. The objective of this study was to determine the influence of static magnetic fields (SMF) on human satellite cell cultures as one of the preferred stem cell sources in skeletal muscle tissue engineering. Experiments were performed using human satellite cells with and without SMF stimulation after incubation with a culture medium containing low [differentiation medium (DM)] or high [growth medium (GM)] concentrations of growth factors. Proliferation analysis using the alamarBlue ${ }^{\circledR}$ assay revealed no significant influence of SMF on cell division. Real-time RT-PCR of the following marker genes was investigated: myogenic factor 5 (MYF5), myogenic differentiation antigen 1 (MYOD1), myogenin (MYOG), skeletal muscle $\alpha 1$ actin (ACTA1), and embryonic (MYH3), perinatal (MYH8) and adult (MYH1) skeletal muscle myosin heavy chain. We detected an influence on marker gene expression by SMF in terms of a down-regulation of the marker genes in cell cultures treated with SMF and DM, but not in cell cultures treated with SMF and GM. Immunocytochemical investigations using antibodies directed against the differentiation markers confirmed the gene expression results and showed an enhancement of maturation after stimulation with GM and SMF. Additional calculation of the fusion index also revealed an increase in
\end{abstract}

Correspondence to: Dr Jens Stern-Straeter, Department of Otolaryngology, Head and Neck Surgery, Medical Faculty Mannheim, University Hospital Mannheim, D-68135 Mannheim, Germany

E-mail: jens.stern-straeter@umm.de

${ }^{*}$ Contributed equally; ${ }^{* *}$ Shared senior authorship

Key words: static magnetic fields, real-time PCR, skeletal muscle, satellite cells, differentiation myotube formation in cell cultures treated with SMF and GM. Our findings show that the effect of SMF on the process of differentiation depends on the growth factor concentration in the culture medium in human satellite cultures. SMF alone enhances the maturation of human satellite cells treated with GM, but not satellite cells that were additionally stimulated with serum cessation. Therefore, further investigations are necessary before consideration of SMF for skeletal muscle tissue engineering approaches.

\section{Introduction}

Generation of functional muscle tissue in vitro using the regenerative potential of stem cells is the ambitious objective of skeletal muscle tissue engineering. The surgical treatment of large muscle defects still represents a demanding practice due to the difficulty of obtaining sufficient amounts of substitute tissue for functional restoration. Thus, in this context, tissue engineering is an encouraging option.

Satellite cells, also called myoblasts, are currently one of the preferred stem cells for skeletal muscle tissue engineering applications, because they can easily be harvested from muscle biopsies, have been extensively characterized, and show a stable differentiation potential into multinucleated myofibers (1). However, it still remains a challenging procedure to induce a perpetual maturation process in expanded satellite cell cultures, a prerequisite to generate sufficient amounts of 'neotissue' for subsequent autologous transplantation. To overcome this obstacle it is important to develop potent differentiation techniques, which should be feasible for a later clinical application, i.e. the absence of possible mutagenic substances or methods that bear long-term risks (e.g. Matrige ${ }^{\circledR}$ ).

Static magnetic fields (SMF) with an intensity of $80 \pm 5 \mathrm{mT}$ have been shown to promote myogenic cell differentiation in the immortal rat cell line L6 in terms of increased accumulation of actin and myosin and formation of large multinucleated myotubes (2). SMF are easily applicable in a cell culture setting and are not genotoxic or oncogenic and therefore, would be practical in clinical applications for skeletal muscle tissue engineering (3). However, it remains unclear whether SMF have an effect on gene expression in myoblasts. Thus, the objective of this study was to analyze the effect of SMF on human satellite cell cultures regarding proliferation and differentiation. 
To decipher the effect of SMF on gene expression, we performed quantitative reverse transcription polymerase chain reaction (qRT-PCR) of well-defined marker genes in human satellite cell cultures treated with medium containing a low concentration of growth factors [differentiation medium (DM)]. Satellite cells treated with a medium containing higher amounts of growth factors [growth medium (GM)] served as the control.

Recent data demonstrate that the reference genes, peptidylprolyl isomerase A (cyclophilin A, PPIA) and the normalization factor (NF) calculated by the geNorm software are suitable endogenous control genes for relative gene quantification profiling studies in human myoblast cell cultures treated with SMF (4). Hence, all qRT-PCR were normalized with the NF.

In addition we used marker genes to identify early stages of muscle development, i.e. myogenic factor 5 (MYF5), myogenic differentiation antigen 1 (MYOD1), and myogenin (MYOG), factors of the myogenic regulator factor (MRF) family, which act as promoters of multiple muscle-specific genes that control the fusion of mononucleated myoblasts into multinucleated myofibers (5). MYF5 enhances muscle cell proliferation and regulates skeletal muscle commitment of myoblasts (6). MYOD1, the myogenic determination factor, is up-regulated during the early stages of myogenesis and promotes cell cycle withdrawal and induction of myoblast differentiation. MYOG is up-regulated during later stages of myogenesis than MYOD1 and acts more specifically during the development of multinucleated myofibers (7). Thus, these genes can be used as distinct markers of differentiation.

One of the most abundant proteins in a mature myotube is the myosin heavy chain (MYH) that exists in various isoforms and accounts for muscle contraction. MYHs are used to characterize the different existing muscle fibers in mature skeletal muscle tissue due to the fact that each fiber type contributes to a variety of functional capabilities (8). Additionally, during myogenesis different developmental isoforms of $\mathrm{MHCs}$ [embryonic (MYH3), perinatal (MYH8) and adult (MYH1)] exist, which can be used as indicators for maturation. Moreover, in vitro experiments with weak SMF (0-200 $\mu \mathrm{T})$ have shown to accelerate $\mathrm{Ca}^{2+} /$ calmodulin-dependent myosin light chain phosphorylation $(9,10)$, and moderate-intensity of SMF (20 mT) stimulation on the rat diaphragm muscle revealed specific changes on ion transporters, which induced bioelectrical and biomechanical alterations (10).

Furthermore, it has recently been demonstrated that SMF stimulation results in actin accumulation in cultured cells and leads to an increase in actin content in myotubes (2), as well as to hypertrophy. In this context it is of high interest to analyze the effect of SMF on the expression of the gene and protein $\alpha 1$ actin (ACTA1) as part of the contractile apparatus in skeletal muscles, which can act as a differentiation marker for the final stage of myogenesis. Therefore, we performed qRT-PCR of human myoblast cell cultures treated with GM or DM for 1, 4, 8, 12 and 16 days to examine the expression of the genes of interest (GOI), MYF5, MYOG, MYH1, MYH3, MYH8 and ACTA1, with or without SMF stimulation with the intensitiy of $80 \mathrm{mT}$ generated by custom-made magnets.

This study presents data about the effects of SMF on proliferation, and gene and protein expression in human satellite cell cultures after identification and verification of valid reference genes, which may be of interest for skeletal muscle tissue engineering approaches.

\section{Materials and methods}

Cell culture. The study protocol was approved by the Ethics Committee of the Medical Faculty Mannheim of the University of Heidelberg, Germany. All patients gave informed written consent. Muscle tissue from 15 patients was harvested during head and neck surgery. Myoblasts (satellite cells) were extracted from minced muscle tissues by digestion with collagenase $\mathrm{B}$ (Roche, Mannheim, Germany) for $60 \mathrm{~min}$ and $0.05 \%$ trypsin$0.02 \%$ EDTA (PromoCell, Heidelberg, Germany) for $45 \mathrm{~min}$ at $37^{\circ} \mathrm{C}$, filtered by a sterile $70 \mu \mathrm{m}$ cell strainer (BectonDickinson, Franklin Lakes, NJ, USA), and purified with the pre-plating technique (11). Primary myoblasts were pooled from the 15 donors and expanded until passage three. Purity of myoblast cultures ( $>80 \%)$ was evaluated by anti-desmin immunostaining. Cells were grown in $0.2 \%$ gelatine-coated culture flasks (Sigma, Deisenhofen, Germany) in Ham's F-10 growth medium, containing $1 \%$ penicillin/streptomycin/ fungizone solution (PSF), 2 mM L-glutamine (PromoCell) and $10 \%$ fetal bovine serum (FBS; PAA Laboratories, Linz, Austria). This medium is referred to as growth medium (GM). Satellite cells were induced to differentiate by incubation with differentiation medium (DM) consisting of minimal essential medium (PromoCell) supplemented with 2\% horse serum (PAA Laboratories), $2 \mathrm{mM}$ L-glutamine, and PSF (PromoCell). GM and DM were changed every $72 \mathrm{~h}$, and cells were cultured at $37^{\circ} \mathrm{C}$ in a humidified atmosphere of $5 \% \mathrm{CO}_{2}$ and $95 \%$ air for $1,4,8,12$ or 16 days.

SMF exposure. SMF were produced by $4 \times 4 \mathrm{~cm}$ neodymium magnetic plaques with a magnetic field of $80 \pm 5 \mathrm{mT}$, verified by a gaussmeter. The magnetic plaques were placed underneath the culture dishes with $1 \mathrm{~mm}$ distance from the cell monolayer culture as described by Coletti et al (2). The magnetic fields were axial with the magnetic North vector crossing the cells (the pole of the magnet which would be repulsed by the Earth's North magnetic pole). Control samples were simultaneously grown and induced to differentiate in identical experimental conditions without the presence of SMF. Magnets did not produce temperature variation.

alamarBlue $^{\circledR}$ (Invitrogen, Darmstadt, Germany) proliferation assay. Satellite cells were cultured in $0.2 \%$ gelatine-coated 96-well plates at a density of 5,000 cells/well in GM or DM. Proliferation was measured on Days $1,4,8,16$ and 21 by measurements of the fluorescence at a wavelength of $540 \mathrm{~nm}$ (exitation) and $590 \mathrm{~nm}$ (emission). Absorbance was monitored at $590 \mathrm{~nm}$.

DNA fragmentation assay. Detection of apoptotic cells was performed using the fluorometric DNA fragmentation detection kit II (BrdU) (PromoKine, Heidelberg, Germany) according to the manufacturer's protocol. Analysis was conducted by fluorescence microscopy of human satellite cell cultures on Days 1, 4, 8,16 and 21 with or without SMF, nourished with DM or GM.

Immunocytochemistry. Immunocytochemical characterization was carried out with cells grown on chamber culture slides 
(Falcon, BD Biosciences, Franklin Lakes, NJ). To indicate the differentiation of myoblasts, the cells were incubated with antibodies directed against: desmin (1:100; Dako, Hamburg, Germany), MYF5, MYOG (1:50; both from Santa Cruz Biotechnology, Heidelberg, Germany), MYH1 (1:20; Santa Cruz Biotechnology), MYH8 (1:40; Abcam, Cambridge, UK) or ACTA1 (1:50; Zymed Laboratories, Invitrogen, Karlsruhe, Germany) followed by the use of a corresponding biotinylated secondary antibody and a streptavidin-biotin horseradish peroxidase complex. The peroxidase reaction was visualized using aminoethylcarbazol (Dako) as a chromogen. For negative controls, the first antibody was omitted. Endogenous peroxidase was blocked with $0.3 \%$ hydrogen peroxide (30 min). Sections were washed with PBS and incubated with normal sheep serum in PBS (30 min, room temperature) to block the non-specific antibody reaction. Cells of five randomly selected fields were counted and averaged independently by two investigators. Nuclei were counterstained with Harris hematoxylin. Light microscopic investigations were performed using a Zeiss Axiophot microscope (Carl Zeiss, Jena, Germany).

Fusion index determination. To quantify differentiation, the number of nuclei in ACTA1, MYH1 or MYH8 positive myotubes was counted and expressed as percentage of the total number of nuclei analyzed. The fusion index (FI) was determined on Days 4, 8, 12 and 16 of culture by dividing the number of nuclei in ACTA1-, MYH1- or MYH8-positive myotubes (with two or more nuclei) by the total number of nuclei $\mathrm{x} 100$.

RNA isolation. Total-RNA was isolated using the RNeasy mini kit (Qiagen, Hilden, Germany), according to the manufacturer's instructions. RNA concentration and purity were determined by A260 and A280 (A260/A280 = 1.7-2.0) measurements using a NanoDrop 8000 spectrophotometer (Thermo Scientific, Schwerte, Germany). Integrity was analyzed by an Agilent 2100 Bioanalyzer (Stratagene-Agilent Technologies, Waldbronn, Germany).

cDNA synthesis and real-time PCR. An aliquot of $0.5 \mu \mathrm{g}$ totalRNA was treated with 1 unit DNase (Fermentas, St. Leon-Rot, Germany) for $30 \mathrm{~min}$ at $37^{\circ} \mathrm{C}$. Reverse transcription of RNA $(0.5 \mu \mathrm{g})$ was performed with oligo(dt) ${ }_{12-18}$ primer, 200 units of SuperScript II (Invitrogen) and 24 units of RiboLock ${ }^{\mathrm{TM}}$ RNase inhibitor (Fermentas) for $1 \mathrm{~h}$ at $42^{\circ} \mathrm{C}$. The cDNA was used for qRT-PCR analysis. All cDNA probes were analyzed for genes of interest: MYF5, NM_005593; MYOG, NM_002479; ACTA1, NM_001100; MYH1, NM_005963; MYH3, NM_002470; MYH8, NM_002472. Moreover, the following reference genes (RG) were investigated: $\beta$-actin (ACTB), NM_001101; $\beta$-2-microglobulin (B2M), NM_004048; glyceraldehyde-3-phosphate dehydrogenase (GAPDH), NM_002046; cyclophilin A (PPIA), NM_203430; large ribosomal protein P0 (RPLP0), NM_001002; TATA box binding protein (TBP), NM_003194. The QuantiTect/Primer assays were purchased from Qiagen. The cDNAs were amplified with Brilliant ${ }^{\circledR}$ II SYBR $^{\circledR}$-Green qRT-PCR Master mix (Stratagene-Agilent Technologies). The thermal profile consisted of 1 cycle at $50^{\circ} \mathrm{C}$ for $2 \mathrm{~min}$ followed by 1 cycle at $95^{\circ} \mathrm{C}(2 \mathrm{~min}), 45$ cycles at $95^{\circ} \mathrm{C}$
(15 sec), $60^{\circ} \mathrm{C}(1 \mathrm{~min})$. Amplification was performed using the Mx3005P ${ }^{\text {TM }}$ qPCR System (Stratagene-Agilent Technologies). For relative quantification, a standard curve was generated in every individual run. Total-RNA was pooled from muscle biopsies of healthy human volunteers. Reverse transcription was performed, and a serial dilution of the cDNA was used to perform the calibration curve. The data was analyzed using the relative standard curve method. For each unknown sample, the relative amount was calculated using linear regression analysis from their respective standard curves. Data were analyzed using Mx3005P analysis software. The efficiencies of all GOI and those of the RGs were calculated in every individual run. Evaluations were performed on Days $1,4,8,12,16$ and 21 by qRT-PCR (in duplicate) in satellite cell cultures nourished with DM and GM and stimulated with or without SMF. Expression results are shown from four independent experiments.

Analysis of expression stability. The qRT-PCR data were analyzed using the Mx3005 $\mathrm{P}^{\mathrm{TM}}$ qPCR system. To compare the stability of six candidate reference genes within sample groups, the software geNorm (12) version 3.4 (Visual Basic application tool for Microsoft Excel) was used according to developer's recommendations.

geNorm uses a gene-stability measurement $\mathrm{M}$, which is defined as the average pairwise variation between a particular gene and all other control genes. It calculates the optimal number of genes necessary for normalization of a target gene and combines them into a NF as previously described by our group and others $(4,12,13)$.

Statistical analyses. The software Sigma Plot was used to carry out statistical analyses by the paired Student's t-test or the Mann-Whitney U test, as well as the Pearson's product correlation test of gene expression among experimental groups. Gene expression results are shown as the mean. A P-value $<0.05$ was considered statistically significant.

\section{Results}

Proliferation. As expected, cell cultures treated with GM revealed higher rates of proliferation than those treated with DM. This proliferation difference was particularly evident during the early days of cell culture. SMF application to human satellite cell cultures incubated with GM or DM did not significantly change the cell turnover, resulting in almost identical proliferation curves over time, irrespective of SMF treatment (Fig. 1).

DNA fragmentation. In cultured human satellite cells, with or without SMF treatment, incubated with GM or DM, apoptotic cells were not seen. These data indicate that SMF did not induce apoptosis at the investigated time points (Days 1, 4, 8, 16 and 21).

RNA quality. RNA samples were examined as to their concentration, purity, and integrity. RNA purity was measured using the NanoDrop ${ }^{\circledR}$ spectrophotometer. Based on the absorbance ratio at 260/280 nm [mean \pm standard deviation (SD), 1.90 \pm 0.05 ], all RNA samples were pure and protein-free. The mean \pm SEM 


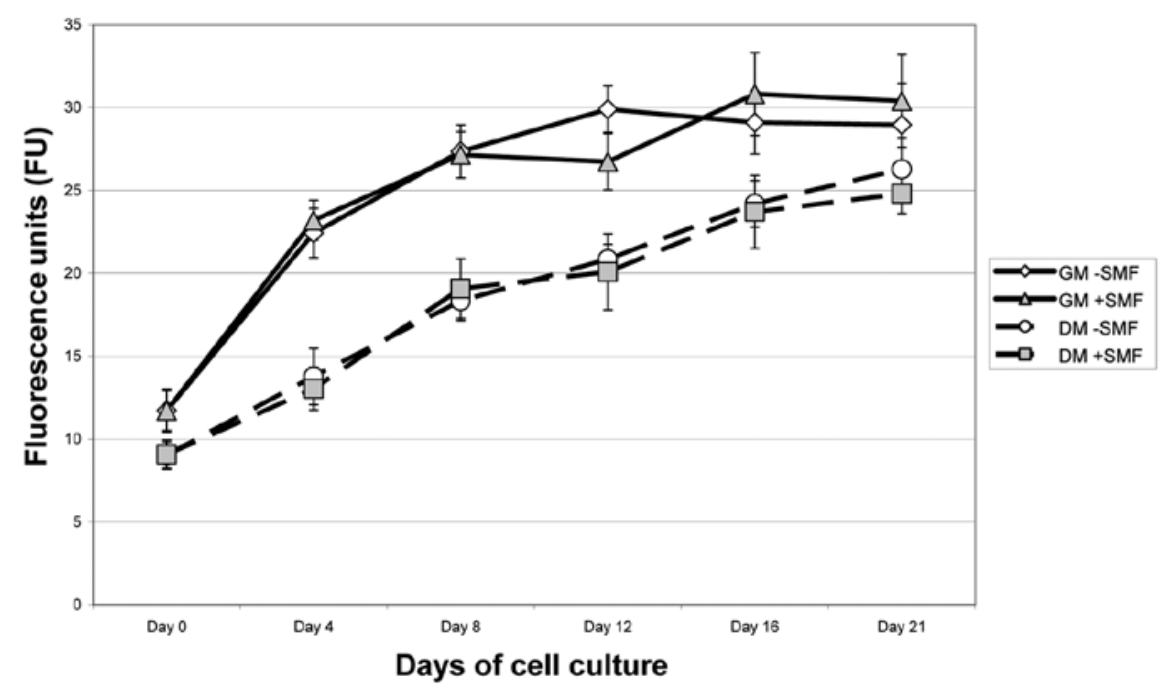

Figure 1. alamarBlue ${ }^{\circledast}$ proliferation assay of human satellite cell cultures treated with or without static magnetic fields (SMF) nourished with growth medium (GM) or differentiation medium (DM).

A260/230 ratio of $1.90 \pm 0.20$ (range from 1.65-2.10) indicated that the RNA was free of phenol and ethanol. RNA integrity was assessed by the calculation of RIN values using the Agilent 2100 Bioanalyzer: RIN values of differentiated and undifferentiated myoblast samples ranged between 7.0 and 8.0.

\section{Gene expression analyses}

MYF5. Under growth conditions without SMF (GM-SMF) the early differentiation marker MYF5 revealed an almost linear decrease in gene expression of about $87 \%$ reaching its lowest relative expression after 21 days (Fig. 2A). In myoblasts cultured under GM with SMF (GM+SMF), after 4 days MYF5 expression was decreased about $45 \%$ in comparison to Day 0, however, this relative expression remained almost stable until Day 16; on Day 21 the MYF5 expression was diminished to about $90 \%$. When comparing GM treatment with or without SMF, it was observed that MYF5 expression was similar with or without SMF stimulation at comparable time points examined. Under differentiation conditions without SMF stimulation (DM-SMF), MYF5 expression decreased time-dependently and almost linearly by 56-95\% between Day 4 and Day 21. Under differentiation conditions with SMF stimulation, MYF5 expression decreased timedependently and almost linearly by $\sim 62-95 \%$ between Day 4 and Day 21. When comparing myoblasts cultured in DM, MYF5 expression was similar with or without SMF stimulation at comparable time points examined. On the other hand, in myoblasts cultured with DM without SMF stimulation, at every time point MYF5 expression was about $47-73 \%$ lower in comparison with myoblasts, cultured in GM without SMF. Finally, in myoblasts cultured in DM with SMF stimulation, at every time point MYF5 expression was about 31-75\% lower in comparison with myoblasts, cultured with GM in the presence of SMF.

MYOD1. Myoblasts cultured with GM without SMF stimulation showed a time-dependent and almost linear increase in MYOD1 gene expression from Day 4 to a peak on Day 12 of
1.9- to 3.3-fold, with a subsequent decrease of $\sim 53$ to $76 \%$ until Day 21 (Fig. 2B). Under growth conditions with SMF stimulation (GM+SMF), myoblasts showed a time-dependent and almost linear increase in MYOD1 gene expression until Day 16 of $\sim 1.8$-fold, which thereafter decreased about $59 \%$ on Day 21. When comparing myoblasts cultured in GM without SMF stimulation to those with SMF stimulation, MYOD1 expression was about 2.4- to 2.8-fold higher (Days 4 to 12) in myoblasts cultured without SMF compared to those with SMF stimulation. Thereafter (on Days 16 and 21), the MYOD1 expression was similar in myoblasts under growth conditions with or without SMF stimulation. Under differentiation conditions without SMF stimulation (DM-SMF), MYOD1 expression was about $50 \%$ lower in comparison to the beginning, however the expression almost linearly increased until Day 12 to levels similar to the beginning, thereafter decreasing about 30\% until Day 21. In myoblasts cultured with DM with SMF stimulation, MYOD1 expression was 1.6- to 1.7 -fold higher (Days 4 and 8) compared to myoblasts at the beginning of the experiment (Day 0). Between Days 8 and 21 MYOD1 expression almost linearly decreased by $\sim 65 \%$. In myoblasts cultured with DM with SMF stimulation, MYOD1 expression was about 1.8- to 3.4-fold higher than in myoblasts cultured with DM but without SMF stimulation. Thereafter, at identical time points the MYOD1 expression was similar in myoblasts under differentiation conditions with or without SMF stimulation. In myoblasts cultured with GM without SMF stimulation (GM-SMF), MYOD1 expression was 1.7- to 3.8-fold higher (Day 4, 8 or 16) in comparison to myoblasts cultured with DM without SMF (DM-SMF). On Day 21 the MYOD1 expression was similar in myoblasts cultured with GM or DM. In GM nourished myoblast cultures stimulated with SMF a timedependent, almost linear increase in the MYOD1 expression until Day 16 was detected, which was about 22-56\% higher on Days 12 and 16 and about 30-59\% lower on Days 4 and 8 in comparison to the expression levels of myoblasts cultured with DM and additionally stimulated with SMF. This group showed a time-dependent decrease after stimulation with SMF to about $43 \%$ on Day 21 in comparison to Day 0. On Day 21 
A

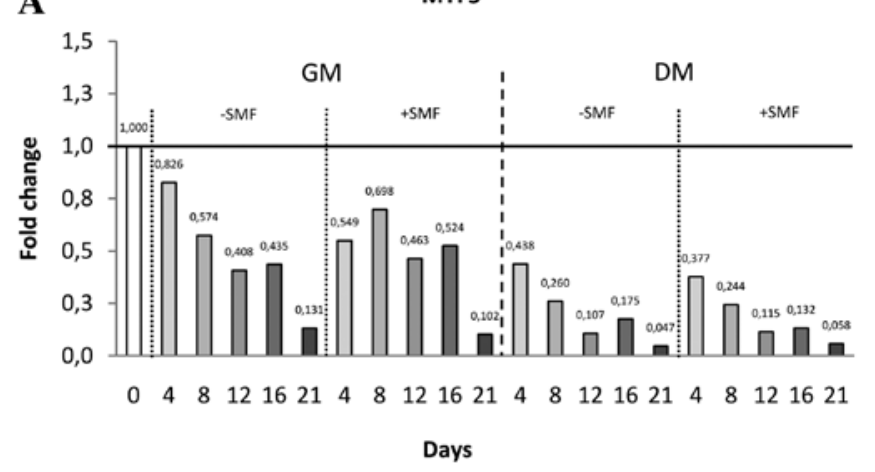

$\mathbf{B}$

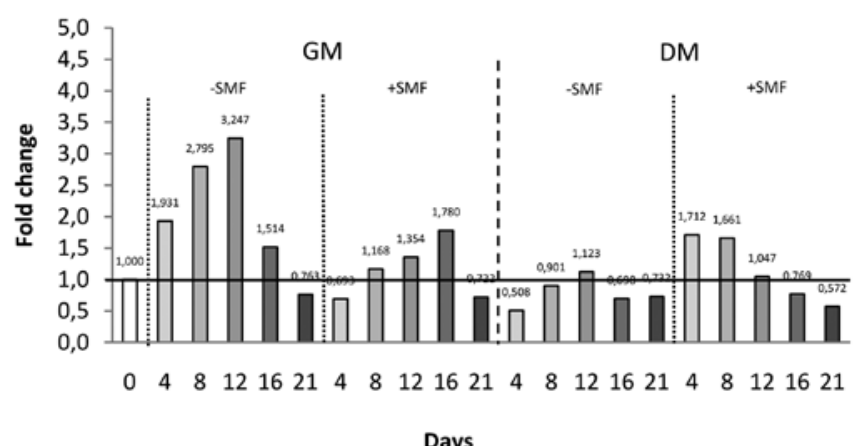

C

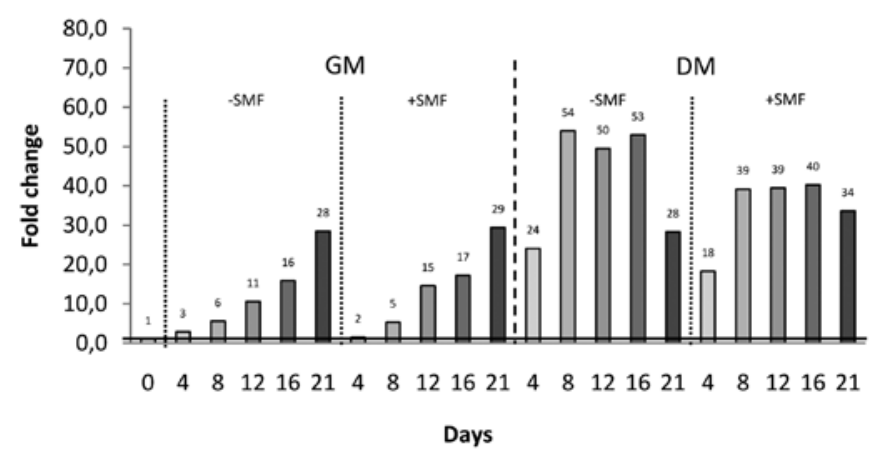

Figure 2. Gene expression analyses (qRT-PCR) of (A) MYF5, (B) MYOD1, (C) MYOG, (D) MYH3, (E) MYH8, (F) MYH1 and (G) ACTA1 of human satellite cell cultures incubated with GM or DM with or without stimulation with static magnetic fields (SMF). The genes were normalized using the normalization factor (NF) calculated by the software geNorm.
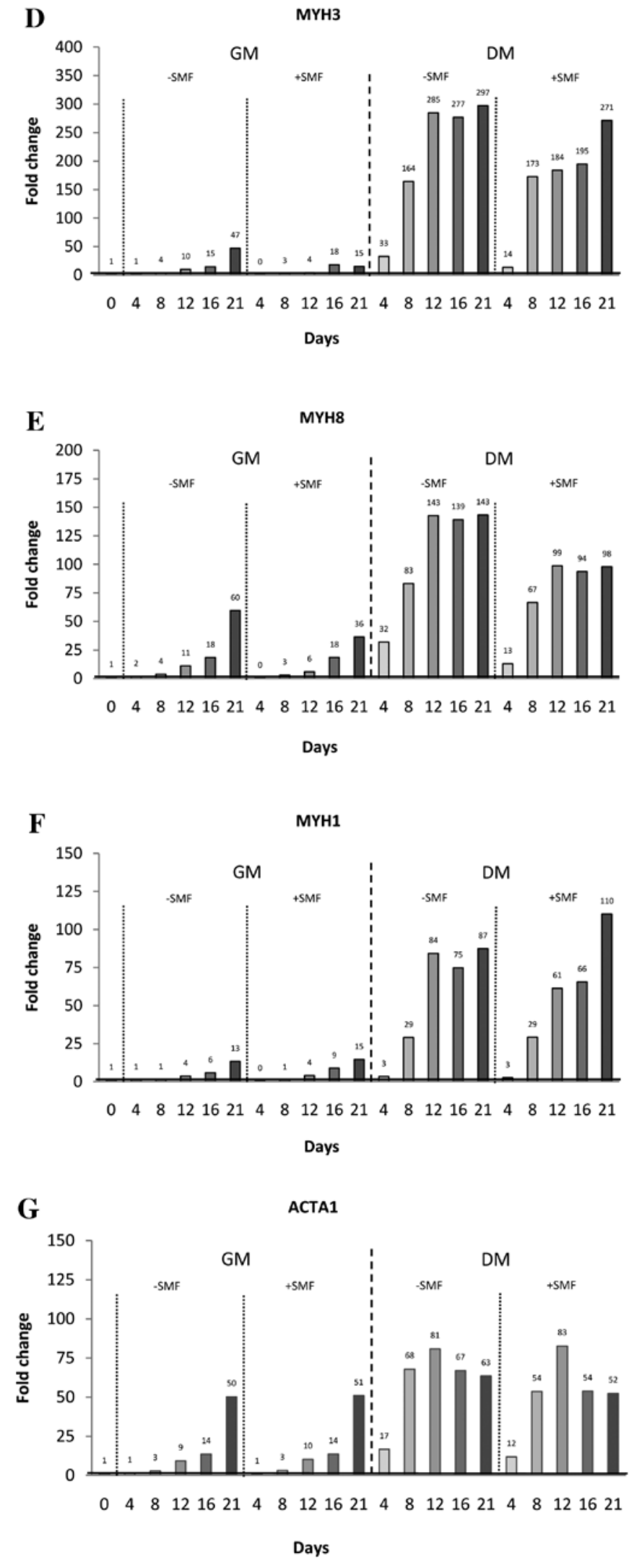

increase in MYOG gene expression between Days 4 and 21 (compared to the expression on Day 0) (Fig. 2C). Under growth conditions with SMF stimulation (GM+SMF), myoblasts showed a time-dependent and almost linear 29-fold increase in MYOG gene expression until Day 21 (compared to the
MYOG. Myoblasts cultured with GM without SMF stimula-

tion revealed a time-dependent and almost linear 3- to 28 -fold the expression of MYOD1 in both SMF-stimulated groups was similar. 
expression on Day 0). In myoblasts cultured in GM, MYOG expression was similar with or without SMF stimulation at comparable time points. Under differentiation conditions without SMF (DM-SMF) stimulation, MYOG expression increased considerably from Day 4 (24-fold) to 16 (53-fold) in comparison to Day 0 and dropped about $47 \%$ on Day 21. In myoblasts cultured with DM with SMF stimulation, MYOG expression was 18- to 40-fold higher (Day 4 until 16) compared to myoblasts at the beginning of the experiment (Day 0). On Day 21, MYOG expression decreased about $15 \%$ in comparison to Day 16. In myoblasts cultured in DM with SMF stimulation, MYOG expression was about 25\% (Day 4 and 16) lower than in myoblasts cultured with DM but without SMF stimulation. Thereafter, at identical time points the MYOG expression was almost similar in myoblasts under differentiation conditions with or without SMF stimulation (28- to 34-fold on Day 21 in comparison to Day 0). Comparison of myoblasts cultured with GM without SMF stimulation to those cultured with DM without SMF, MYOG expression was considerably lower $(-88 \%)$ on Day 4 and until Day $16(-70 \%)$ in comparison to myoblasts cultured with DM at comparable time points. On Day 21 the MYOG expression was similar in myoblasts nourished with GM or DM. Finally, in myoblasts cultured in DM with SMF stimulation, at every time point MYOG expression was about 1.2- to 9-fold higher in comparison to myoblasts cultured with GM and SMF stimulation.

MYH3. Myoblasts cultured in GM without SMF stimulation showed a time-dependent 2 - to 47 -fold increase in MYH3 gene expression between Days 4 and 21 (compared to the expression on Day 0) (Fig. 2D). Myoblasts cultured in GM with SMF stimulation showed a time-dependent increase in MYH3 gene expression until Day 16 (compared to the expression on Day 0), with a marginal decrease of $17 \%$ on Day 21 in comparison to Day 16. Comparison of myoblasts cultured with GM with vs. without SMF stimulation, revealed that the MYH3 expression was similar with or without SMF stimulation until Day 16. On Day 21, cells cultured without SMF showed a 47-fold augmented MYH3 expression (relative to Day 0) in comparison to a 15 -fold increased in MYH3 expression in myoblasts with SMF stimulation. In myoblasts cultured with DM without SMF stimulation, MYH3 expression showed a considerable 33- to 285-fold increase starting on Day 4 until Day 12 (compared to the expression on Day 0), which almost remained constant until Day 21. Under differentiation conditions with SMF stimulation (DM+SMF), MYH3 expression was 14- to 173-fold higher on Day 4 and 8, thereafter timedependently increased to 271-fold on Day 21 (compared to the expression on Day 0). When comparing myoblasts nourished with DM with vs. without SMF stimulation, MYH3 expression was about 2.4- to 1.4-fold higher (Days 4 and 16) in those without than in myoblasts cultured with SMF stimulation with the exception on Day 8, where the expression was almost similar in both groups, i.e. with or without SMF stimulation. Furthermore, comparison of myoblasts cultured in DM without SMF stimulation to those cultured in GM without SMF, revealed that at every time point MYH3 expression was about 6.3- to 41-fold higher in myoblasts culuted in DM than in those nourished with GM. Finally, comparison of myoblasts treated with SMF stimulation in GM vs. DM showed that
SMF stimulated myoblasts cultured with DM showed a 14- to 58 -fold higher expression at every time point than myoblasts cultured in GM with SMF.

MYH8. Myoblasts under growth conditions without SMF stimulation showed a time-dependent about 2- to 60-fold increase in MYH8 gene expression starting on Day 4 and until Day 21 (compared to the expression on Day 0) (Fig. 2E). Myoblasts cultured with GM and stimulated with SMF demonstrated a time-dependent and almost linear 3- to 36-fold increase in MYH8 expression on Day 8 and until Day 21 (compared to the expression on Day 0). Comparing myoblasts cultured with GM with or without SMF stimulation, MYH8 expression was almost similar until Day 16. Thereafter, on Day 21, the MYH8 expression was 1.7-fold higher in GM cultured cells without SMF stimulation in comparison to myoblasts with SMF stimulation. Under differentiation conditions (DM) myoblasts cultured without SMF stimulation showed a 32- to 143-fold increase in MYH8 expression on Day 4 and until 12 (compared to the expression on Day 0), which thereafter remained almost constant until Day 21. Myoblast cultured in DM and stimulated with SMF showed a 13- to 98-fold increase on Days 4 to 12 (compared to the expression on Day 0), which thereafter remained almost constant until Day 21. A comparison of myoblasts nourished with DM with vs. without SMF stimulation, revealed that at all time points examined MYH8 expression was about 1.5- to 2.4-fold higher in myoblasts without than with SMF stimulation. Furthermore, comparison of myoblasts treated without SMF stimulation in GM vs. DM revealed that myoblasts cultured with DM without SMF stimulation showed a 2.4- to 21-fold increased MYH8 expression at all time points examined in comparison to GM-nourished myoblasts. Finally, a comparison of myoblasts treated with SMF stimulation in GM vs. DM, demonstrated that SMF stimulation resulted in higher MYH8 expression at all investigated time-points in cell cultures treated with DM in comparison to GM-nourished cultures. The expression of the MYH8 gene in myoblasts treated with DM+SMF was 2.7- and 22.3-fold higher on Day 21 and Day 8, respectively than in those treated with GM+SMF.

MYH1. Myoblasts cultured with GM without SMF stimulation demonstrated a time-dependent, almost linear 1- to 13-fold increase in MYH1 gene expression on Day 4 until 21 (compared to the expression on Day 0) (Fig. 2F). In myoblasts cultured under growth conditions with additional SMF stimulation the differentiation marker MYH1 showed an almost linear 1- to 15-fold increase in gene expression on Day 8 until 21 (compared to the expression on Day 0). A comparison of of MYH1 expression in myoblasts cultured in GM with our without SMF stimulation, showed that MYH1 expression was similar with or without SMF stimulation at comparable time points. Under differentiation conditions without SMF stimulation, myoblasts showed a 3- to 84-fold augmentation in MYH1 gene expression on Day 4 until Day 12 (compared to the expression on Day 0), which remained almost constant until Day 21. Myoblasts cultured in DM and with SMF stimulation showed a time-dependent, almost linear 3- to 110-fold increase in the MYH1 expression on Day 4 until 21 (compared to the expression on Day 0 ). We then compared myoblasts cultured in 
DM in the presence or absence of SMF. In myoblasts cultured with DM, MYH1 expression was similar with or without SMF stimulation on Days 4 and 8, however, on Days 12 and 16 MYH1 expression was 1.1- to 1.4-fold higher in myoblasts with SMF stimulation in comparison to cells without SMF stimulation, and on Day 21, MYH1 expression was 1.3-fold higher in cells with SMF compared to cells without SMF stimulation.Furthermore, a comparison of myoblasts treated with GM vs. DM without SMF was performed. At all time points under test, myoblasts cultured with DM without SMF revealed 3- to 87-fold increased expression rates of the MYH1 gene in comparison to GM nourished myoblasts. Finally, we compared treatment with GM vs. DM in the presence of SMF stimulation. At all time points under test, myoblasts cultured in DM and stimulated with SMF demonstrated a 3- to 29-fold increased expression rate of the MYH1 gene in comparison to myoblasts cultured with GM.

ACTA1. Myoblasts cultured with GM without SMF stimulation showed a time-dependent and almost linear 1- to 50-fold increase in ACTA1 gene expression on Day 4 until 21 (compared to the expression on Day 0) (Fig. 2H). Under growth conditions, myoblasts stimulated with SMF showed a time-dependent and almost linear 1- to 51-fold increase of the ACTA1 expression on Day 4 until 21 (compared to the expression on Day $0)$. We then compared treatment of myoblasts with GM with vs. without SMF stimulation. In myoblasts cultured with GM, ACTA1 expression was similar with or without SMF stimulation at comparable time points. In myoblasts cultured with DM without SMF stimulation, ACTA1 expression increased 17-fold on Day 4 and 81-fold on Day 12 (compared to the expression on Day 0) and, thereafter, ACTA1 expression declined about $35 \%$ on Day 16 and, additionally, 6\% on Day 21. Myoblasts cultured with DM and stimulated with SMF demonstrated an almost linear 12- to 83-fold increase in ACTA1 expression on Day 4 until 12 compared to the expression on Day 0 . Then the expression declined around $35 \%$ on Day 16 and remained stable at 54-fold (Day 16) and 52-fold (Day 21) compared to the initial expression on Day 0. Furthermore, SMF stimulated myoblast cultures nourished with DM, showed at almost all investigated time points lower expression rates than myoblasts cultured in DM but not stimulated with SMF. The ACTA1 expression was between 21\% (Day 8) and 29\% (Day 4) lower in myoblasts treated with SMF compared to non-SMF stimulated cell cultures. On Day 12 the expression value was similar. We next compared treatment of myoblasts with GM vs. DM without SMF stimulation. Non-SMF stimulated myoblasts that were treated with DM showed at every time point a higher expression that ranged beteen 1.3-fold on Day 21 to 22.7-fold on Day 8 compared to myoblasts cultured with GM. Finally, we compared treatment of myoblast with GM vs. DM with SMF stimulation. In myoblasts cultured with DM with SMF stimulation, until Day 16 ACTA1 expression was about 3.9-fold (Day 16) to 18-fold (Day 8) higher in comparison with myoblasts, cultured with GM and SMF stimulation. On Day 21 the expression rates were equal between the two groups.

Immunocytochemistry. In order to validate the measured mRNA expression and detect possible differences with the corresponding protein levels immunocytochemical staining was performed using monoclonal antibodies directed against MYF5, MYOG, MYH8, MYH1 and ACTA1 (Table I). The amount of cells positively stained with antibodies directed against the early differentiation marker MYF5 showed an increase during the early days of cell culture, followed by a time-dependent constant decrease of expression levels in all groups evaluated. No difference between cultures treated with or without SMF was seen. These findings are in parallel with our gene expression data measured by qRT-PCR. Analyses of the amount of MYOG-immunoreactive cells revealed an increase in cells cultured with DM or GM during the time points examined. In myoblasts cultured with GM and SMF, more positive myotubes were measured on Day 21 in comparison to cell cultures without SMF stimulation. In DM nourished myoblsats, this enhancement of MYOG-positive cells was not observed. The amount of MYH8-immunoreactive cells was higher in myoblasts cultured with GM and SMF in comparison to cells cultured without SMF stimulation during all time points under test. Again, this enhancement effect of SMF was not observed when satellite cells were nourished with DM. Moreover, our qRT-PCR results of MYH8 mRNA expression are in parallel with those found by antibody staining (protein level). In GM nourished cell cultures, the amount of MYH1 immunoreactive cells was increased after SMF treatment in comparison to cells without SMF stimulation. In general, in all groups under test, the increase in the amount of MYH1 immunoreactive cells was almost linear and time-dependent indicating the advancing maturation. In DM cultured myoblasts this effect was not seen. Additionally, in DM-nourished myoblasts, a higher amount of MYH1-immunoreactive cells was measured in the groups without SMF stimulation. ACTA1-immunoreactive myoblasts were found in all experimental groups, starting from Day 8 of analyses. On Days 12 and 21, more ACTA1-positive cells were found after stimulation with SMF in comparison to cells without SMF stimulation in the GM-nourished group. Such an augmentation was not observed in SMF-stimulated cells when nourished with DM.

Immunocytochemical characterization of cultured human myoblasts is representatively shown in Fig. 3: on Day 12, antiMYH8 staining in cultured human satellite cells incubated with GM, demonstrated cytoplasmic (thick filaments of the myofibrils) localization of MYH8 in elongated, multinucleated myotubes. Stronger distribution was found after stimulation with SMF (Fig. 3A and B).

Anti-MYH1 staining of cultured (on Day 12) human myoblasts incubated with GM, showed MYH1 in elongated, multinucleated myotubes. Again more positive cells were found after stimulation with SMF (Fig. 3C and D).

Anti-ACTA1 staining of cultured (on Day 12) human satellite cells incubated with GM showed an enhanced cytoplasmic distribution pattern of the immunoreaction after stimulation with SMF (Fig. 3E and F).

Fusion index. To evaluate the impact of SMF on human satellite cell cultures, an additional, independent and well-accepted method was used, i.e. the fusion index was calculated in forming myotubes expressing ACTA1 and MYH1 at different time points with or without SMF stimulation (Fig. 4). In satellite cells cultured with GM, the fusion index of ACTA1- and MYH1-positive myotubes showed a time-dependent increase 
Table I. Immunocytochemical evaluation of muscle-specific differentiation marker proteins in human satellite cells cultured in growth medium or differentiation medium with or without static magnetic field stimulation from Day 4 until 21.

Staining pattern ${ }^{\mathrm{a}}$

\begin{tabular}{|c|c|c|c|c|c|}
\hline Antibody & Day 4 & Day 8 & Day 12 & Day 16 & Day 21 \\
\hline \multicolumn{6}{|l|}{ GM-SMF } \\
\hline MYF5 & +++ & +++ & ++ & +++ & + \\
\hline MYOG & ++ & ++ & ++ & ++++ & ++++ \\
\hline MYH8 & - & + & + & + & ++ \\
\hline MYH1 & - & ++ & ++ & +++ & +++ \\
\hline ACTA1 & - & ++ & ++ & +++ & +++ \\
\hline \multicolumn{6}{|l|}{ GM+SMF } \\
\hline MYF5 & +++ & +++ & +++ & ++ & + \\
\hline MYOG & ++ & +++ & +++ & +++ & ++++ \\
\hline MYH8 & ++ & +++ & ++ & +++ & +++ \\
\hline MYH1 & - & ++ & +++ & +++ & ++++ \\
\hline ACTA1 & - & ++ & +++ & +++ & ++++ \\
\hline \multicolumn{6}{|l|}{ DM-SMF } \\
\hline MYF5 & +++ & +++ & ++ & ++ & + \\
\hline MYOG & ++ & +++ & +++ & ++++ & ++++ \\
\hline MYH8 & ++ & +++ & +++ & ++++ & ++++ \\
\hline MYH1 & - & +++ & +++ & ++++ & ++++ \\
\hline ACTA1 & - & +++ & +++ & +++ & ++++ \\
\hline \multicolumn{6}{|l|}{$\mathrm{DM}+\mathrm{SMF}$} \\
\hline MYF5 & +++ & +++ & ++ & ++ & + \\
\hline MYOG & ++ & ++ & +++ & +++ & +++ \\
\hline MYH8 & - & +++ & +++ & +++ & +++ \\
\hline MYH1 & - & +++ & +++ & +++ & +++ \\
\hline ACTA1 & - & ++ & +++ & +++ & +++ \\
\hline
\end{tabular}

aPercentage of cells stained by the monoclonal antibodies is symbolized by '++++' (70-100\%), '+++' (50-70\%), '++' (30-50\%), '+' (1-30\%) and '-' (no staining). GM, growth medium; DM, differentiation medium; SMF, static magnetic fields.

with the highest level on Day 21. SMF significantly increased the fusion index of both differentiation markers ACTA1 and MYH1 during all time points of observation in comparison to non-stimulated cells cultured with GM. In DM-nourished cells the fusion index of MYH1 and ACTA1 also increased with time in satellite cells without SMF stimulation, except for ACTA1 on Day 12. After stimulation with SMF no significant difference in the fusion index of ACTA1-positive myotubes was detected in cells cultured with DM. On the contrary, the MYH1 fusion index was higher after stimulation with SMF with a time-dependent increase.

The ACTA1 fusion index showed a time-dependent increase indicating the differentiation of satellite cell cultures nourished with GM with or without SMF stimulation, but in DM treated cultures with or without SMF stimulation the ACTA1 fusion index remained almost at a constant level between 0.1 and 0.2 .

SMF stimulation in GM or DM cultured myoblasts resulted in a constant augmentation of the MYH1 fusion indices with time, however the fusion indices were higher in DM-treated cultures at all examined time-points in comparison to GM-nourished myoblasts.

\section{Discussion}

Identification of strong and clinically applicable differentiation stimuli that can induce the fusion of mononucleated myoblasts into multinucleated myofibers is one important prerequisite to clinical utilization for the emerging field of skeletal muscle tissue engineering. Various stimuli, (e.g. neuronal co-cultivation, gene transfer methods, sarcoma extract Matrige ${ }^{\circledR}$ ) have been investigated in terms of their differentiation potential, but all lack clinical feasibility due to unpredictable risks for the patients (1).

SMF generated by magnets have been shown to promote myogenic cell differentiation and hypertrophy in the immortal L6 rat skeletal muscle cells in terms of increased accumulation of actin and myosin, both proteins of the contractile apparatus (2). Additionally, formation of larger myotubes was observed by others after treatment with $80 \mathrm{mT}$ magnetic fields. Furthermore, 

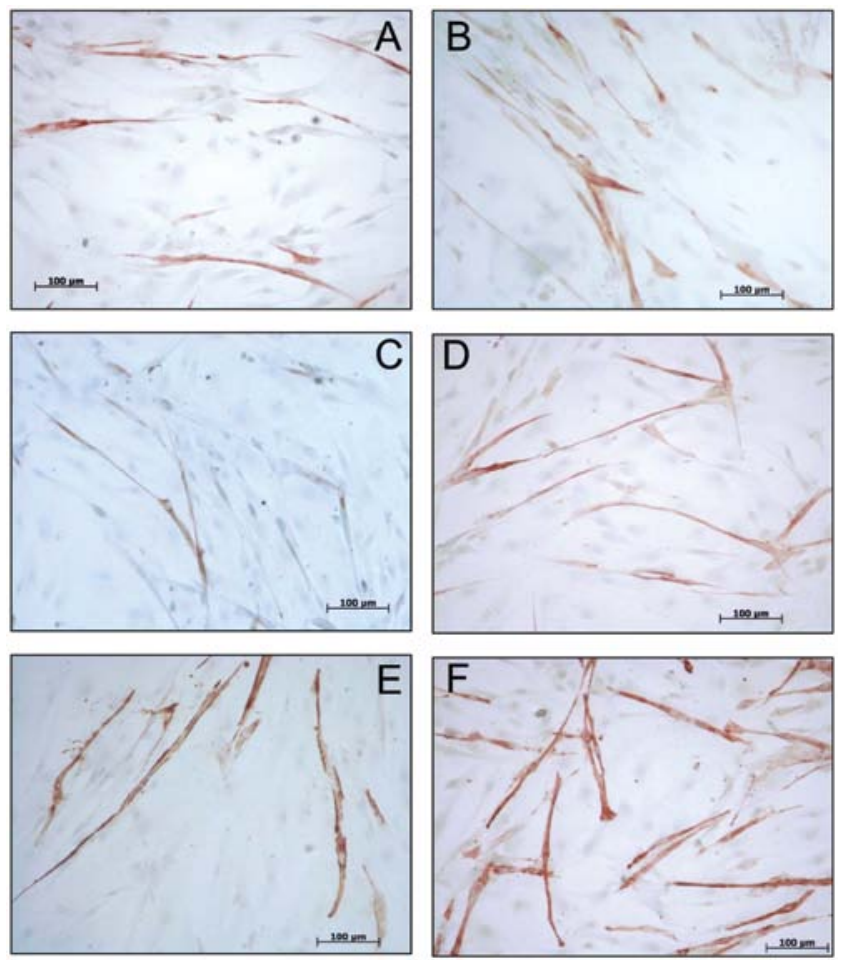

Figure 3. Immuncytochemical characterization of human satellite cell cultures. Anti-MYH8 staining on Day 12 in satellite cell cultures incubated (A) with GM and (B) with GM and SMF stimulation. Anti-MYH1 staining on Day 12 in satellite cell cultures incubated with (C) GM and (D) with GM and SMF stimulation. Anti-ACTA1 staining on Day 12 in satellite cell cultures incubated with (E) GM and (F) with GM and SMF stimulation.

an accelerated differentiation in myoblasts stimulated with a $50 \mathrm{mT}$ magnetic field was described, when microparticles were introduced into the cytoplasm (14). Additionally, the authors report that differentiation marker expression was detectable at early time points in myoblasts containing the microparticles after SMF stimulation. SMF are already frequently applied on human skeletal muscles for musculoskeletal pain relief and until now no oncogenic or genotoxic effects were observed in living cells (3). Based on these observations, we investigated the impact of SMF as a differentiation stimulus on human satellite cells regarding proliferation and gene and protein expression in human satellite cell cultures incubated with a medium containing low concentration of growth factors (DM) with or without SMF treatment. Human satellite cells incubated with a medium containing high amounts of growth factors (GM) with or without SMF stimulation served as a control group, since differentiation of skeletal myoblasts in cultures is negatively regulated by growth factors in the serum, indicating that signaling pathways induced by growth factors present in serum will facilitate myoblast proliferation but inhibit myogenesis (6).

Proliferation analysis using the alamarBlue ${ }^{\circledR}$ assay revealed no differences between human satellite cells with or without SMF stimulation, independent from the growth factor concentration in the culture medium, supporting the data of others showing that SMF does not significantly affect cell proliferation of myoblasts (2). These data are supported by the fact that apoptotic cells were not seen after stimulation with SMF. In general, it is noteworthy to mention that the effect of SMF on cell proliferation seems to be inversely proportional to the strength of the magnetic field. Furthermore, it has been reported that prolonged exposure to the strong magnetic field of $7 \mathrm{mT}$ inhibits the growth of the human tumor cell lines HTB63 (melanoma), HTB 77IP3 (ovarian carcinoma) and CCL86 (lymphoma) $(3,15)$. Thus, the effect of SMF varies depending on the cell type and strength of the magnetic field. As expected, satellite cell cultures treated with GM showed higher proliferation rates in the beginning in comparison to satellite cells nourished with DM, which can be explained by the different concentrations of growth factors in the culture media. High concentrations of growth factors will facilitate myoblast proliferation, but inhibit differentiation (6).

Satellite cell differentiation is initiated by an up-regulation of the myogenic regulator protein MYF5, which is necessary for proliferation and specialization followed by a downregulation with the ongoing differentiation process (16) and can therefore be used as an early marker of myogenesis. We observed no significant effect of SMF stimulation on MYF5 expression in human satellite cell cultures. All groups showed

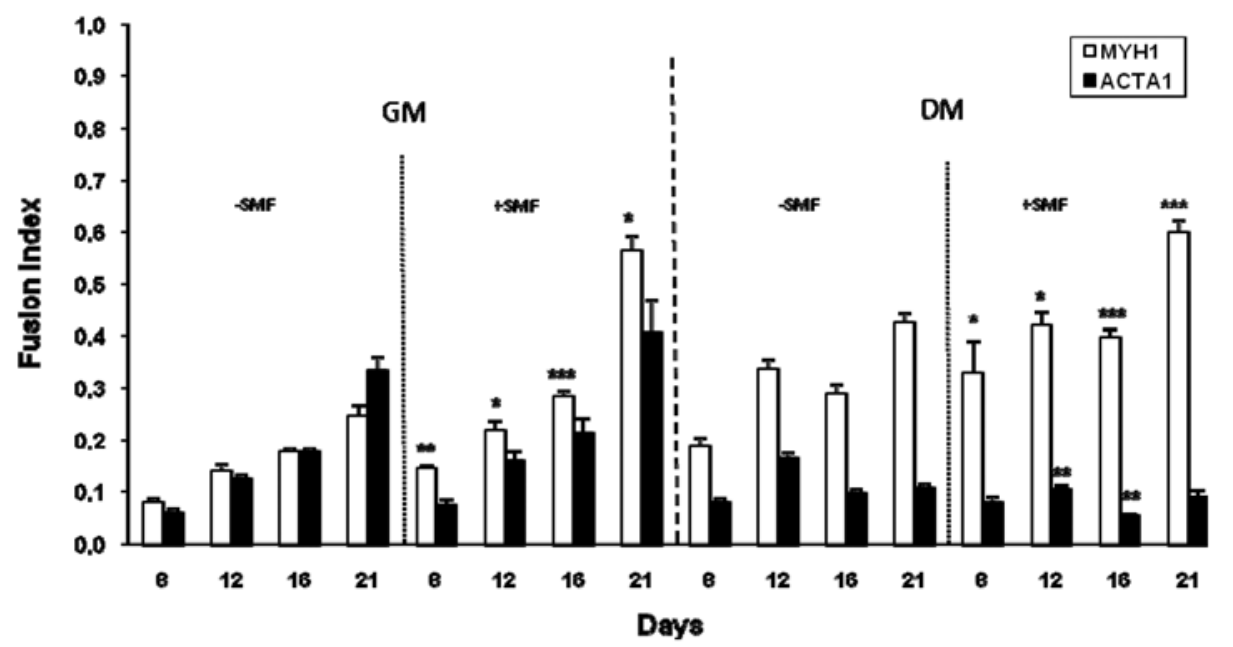

Figure 4. Fusion index in MYH1 or ACTA1 stained cells cultured in growth medium (GM) or differentiation medium (DM) with (+SMF) or without (-SMF) static magnetic field stimulation. 
a peak expression of MYF5 on Day 4, followed by consecutive declines, supporting the assumption of the beginning of differentiation of all cells and verifying the myogenic cell line character. Additionally, our gene expression results were confirmed by the protein expression of MYF5 underlying the high degree of accuracy. Therefore, it is suggested that SMF does not inhibit differentiation and proliferation during the early course of myogenesis.

MYOD1, a transcription factor which promotes several muscle specific genes by heterodimerization with 'E-proteins' and binding to the DNA-sequence CANNTG, induces differentiation by withdrawal of the cell-cycle both by cross-talking with the cell cycle regulators (6) and by inhibiting cytokine signals (17). The up-regulation of MYOD1 represents the start of the myogenic differentiation process. In cell cultures nourished with GM, we found a time-dependent increase until Day 16 in cells with SMF stimulation, myoblasts without SMF stimulation showed a 2.4- to 2.8-fold higher expression of MYOD1 with a peak expression on Day 12. In human satellite cell cultures treated with DM and SMF, higher concentrations of MYOD1 were measured by qRT-PCR until Day 8, followed by similar expression levels in comparison to cells without SMF stimulation and cultured with DM. In summary, SMF appears to have a mild amplification effect on MYOD1 gene expression during the early courses of myogenesis if human myoblast cells are additionally stimulated by serum cessation. This effect is not evident if cell cultures are cultured with a medium containing high concentrations of growth factors (GM), indicating that the influence of SMF on the MYOD1 expression highly depends on growth factor concentration.

The gene expression results of MYOG, a member of the MRF family, which acts later and more specifically during the development of myofibrils (18) showed a time-dependent increase with the ongoing differentiation in myoblasts cultured in GM, but no evident effect of the SMF stimulation. Interestingly, myoblasts cultured with DM also showed a time-dependent up-regulation with the advancing process of maturation, but lower MYOG gene expression was detected after the stimulation with SMF. In our study, this effect was verified by immunocytochemical investigations, also showing a lower amount of MYOG-positive cells in SMF-stimulated myoblasts after culture with DM. This finding is in contrast to the data of Coletti et al (2), who detected no differences in the MYOG gene expression between SMF-stimulated and nonstimulated L6 cells despite the enhancing maturation. This discrepancy may be explained by the different origin of the cells examined, i.e. an immortal cell line vs. primary human cells, additionally highlighting the importance to utilize primary human cells for tissue engineering research.

MYHs are of the most abundant proteins in a mature myotube, existing in various isoforms, which can be used to characterize fiber type and developmental status (19). During myogenesis, the MYH3 and a MYH8 isoforms can be detected in developing myofibers and therefore, can be used as indicators for maturation (16). In our study, in almost all groups, the embryonic-MYH3 showed a constant increase in concentration as the number of myotubes increased. In satellite cells cultured with GM and SMF stimulation, no substantial differences were observed concerning the MYH3 gene expression. In cell cultures nourished with DM, SMF stimulation led to a decrease of the mRNA concentration at all time points examined until Day 21. This SMF-dependent effect on human satellite cells could also be found concerning the expression of the perinatal-isoform MYH8, which showed decreased mRNA concentrations after SMF stimulation in comparison to cells without SMF stimulation. In myoblasts cultured with GM no amplification effect of the SMF was seen on the gene expression level, but the immunocytochemical staining revealed an increase of MYH8-positive cells after stimulation with SMF. The adult isoform MYH1 was also suppressed after SMF stimulation in myoblsats cultured with DM. This effect was not seen in human satellite cells nourished with GM. The data about the gene expression of the different isoforms of the MYH genes indicate that SMF can suppress an up-regulation in the gene expression and therefore, inhibit differentiation after SMF stimulation if myoblasts are cultured with DM. This effect was not detected in cells cultured with GM, where differences after SMF stimulation were detected. This finding supports the hypothesis that the effect of SMF on the gene expression in human satellite cells depends on the growth factor concentration. The effect of SMF on the expression at the protein level (evaluated by immunocytochemistry) showed an enhancement of MYH-expression in the cells stimulated with GM and SMF and not in cells nourished with DM, i.e. the degree of impact on cell physiology depends on the growth factor concentration in the culture medium. In vitro experiments on skeletal muscle could show that DC-generated SMF in the range of 0-200 $\mu \mathrm{T}$ accelerates $\mathrm{Ca}^{2+} /$ calmodulin-dependent myosin light chain phosphorylation and therefore, influences physiological properties on the contractile apparatus (9).

The ACTA1 gene encodes skeletal muscle $\alpha$-actin, which is a major constituent of the contractile apparatus and along with myosin, is essential for muscle contractions (20). Therefore, ACTA1 expression can be used as a marker of the final stages during myogenesis (21). Our study shows that ACTA1 expression in human satellite cell cultures nourished with DM was decreased after SMF stimulation, indicating a lower degree of maturation after SMF stimulation. In cell cultures nourished with GM this effect was not observed.

According to our immunocytochemical data, we calculated the fusion index as a gene-independent marker of differentiation in ACTA1- or MYH1-positive human satellite cell cultures, showing a statistically significant increase of the fusion index of both differentiation markers after incubation with GM and SMF stimulation. Our findings provide evidence that SMF stimulation enhances maturation of human myoblasts/satellite cells when being incubated with high concentration of growth factors. Interestingly, this effect was not followed by an up-regulation of the examined marker genes. However, a suppression of the marker genes, as detected in satellite cells nourished with DM was not observed. This effect was not seen in human myoblasts, which were stimulated to differentiate by growth factor cessation, giving evidence that the effect of SMF depends on the growth factor concentration in the culture medium. However, the underlying mechanisms of the differentiation process remain unknown to date and will be the subject of further investigations.

In conclusion, we observed a suppression of well-defined marker genes in cultured human myoblasts after stimulation with SMF of $80 \mathrm{mT}$ using qRT-PCR with internationally- 
accepted reference genes for cells incubated in DM. However, SMF enhances the maturation of cultured human satellite cells cultured with GM, indicating that the effect of SMF depends on the growth factor concentration in the culture medium. Therefore, further investigations are necessary before consideration of SMF for skeletal muscle tissue engineering approaches.

\section{Acknowledgements}

The authors would like to thank Mrs. Gabriella Stauch and Mr. Michael Collins for assistance with the manuscript as well as Mrs. Petra Prohaska, Mrs. Ulrike Traut and Mrs. Rebecca Hollender for their excellent technical support.

\section{References}

1. Stern-Straeter J, Riedel F, Bran G, Hormann K and Goessler UR: Advances in skeletal muscle tissue engineering. In Vivo 21: 435-444, 2007.

2. Coletti D, Teodori L, Albertini MC, Rocchi M, Pristera A, Fini M, Molinaro M and Adamo S: Static magnetic fields enhance skeletal muscle differentiation in vitro by improving myoblast alignment. Cytometry A 71: 846-856, 2007.

3. Miyakoshi J: Effects of static magnetic fields at the cellular level. Prog Biophys Mol Biol 87: 213-223, 2005.

4. Stern-Straeter J, Bonaterra GA,Zügel S, Kassner SS, Hörmann K, Kinscherf R and Goessler UR: Evaluation of valid reference genes during stimulation with static magnetic fields in human myoblast cultures. Mol Med Rep 3: 237-243, 2009.

5. Brand-Saberi B: Genetic and epigenetic control of skeletal muscle development. Ann Anat 187: 199-207, 2005

6. Kitzmann M and Fernandez A: Crosstalk between cell cycle regulators and the myogenic factor MyoD in skeletal myoblasts. Cell Mol Life Sci 58: 571-579, 2001.

7. Ridgeway AG, Petropoulos H, Wilton S and Skerjanc IS: Wnt signaling regulates the function of MyoD and myogenin. J Biol Chem 275: 32398-32405, 2000.

8. Wright C, Haddad F, Qin AX and Baldwin KM: Analysis of myosin heavy chain mRNA expression by RT-PCR. J Appl Physiol 83: 1389-1396, 1997.
9. Pilla AA, Muehsam DJ, Markov MS and Sisken BF: EMF signals and ion/ligand binding kinetics: prediction of bioeffective waveform parameters. Bioelectrochem Bioenerg 48: 27-34, 1999.

10. Itegin M, Gunay I, Logoglu G and Isbir T: Effects of static magnetic field on specific adenosine-5'- triphosphatase activities and bioelectrical and biomechanical properties in the rat diaphragm muscle. Bioelectromagnetics 16: 147-151, 1995.

11. Machida S, Spangenburg EE and Booth FW: Primary rat muscle progenitor cells have decreased proliferation and myotube formation during passages. Cell Prolif 37: 267-277, 2004.

12. Vandesompele J, De Preter K, Pattyn F, Poppe B, Van Roy N, De Paepe A and Speleman F: Accurate normalization of real-time quantitative RT-PCR data by geometric averaging of multiple internal control genes. Genome Biol 3: Research0034, 2002.

13. Stern-Straeter J, Bonaterra GA, Hormann K, Kinscherf R and Goessler UR: Identification of valid reference genes during the differentiation of human myoblasts. BMC Mol Biol 10: 66, 2009.

14. Yuge L and Kataoka K: Differentiation of myoblasts is accelerated in culture in a magnetic field. In Vitro Cell Dev Biol Anim 36: 383-386, 2000.

15. Raylman RR, Clavo AC and Wahl RL: Exposure to strong static magnetic field slows the growth of human cancer cells in vitro. Bioelectromagnetics 17: 358-363, 1996.

16. Cosgrove BD, Sacco A, Gilbert PM and Blau HM: A home away from home: challenges and opportunities in engineering in vitro muscle satellite cell niches. Differentiation 78: 185-194, 2009.

17. Kataoka Y, Matsumura I, Ezoe S, Nakata S, Takigawa E, Sato Y, Kawasaki A, Yokota T, Nakajima K, Felsani A and Kanakura Y: Reciprocal inhibition between MyoD and STAT3 in the regulation of growth and differentiation of myoblasts. J Biol Chem 278: 44178-44187, 2003.

18. Sassoon DA: Myogenic regulatory factors: dissecting their role and regulation during vertebrate embryogenesis. Dev Biol 156: 11-23, 1993.

19. Pette D and Staron RS: Transitions of muscle fiber phenotypic profiles. Histochem Cell Biol 115: 359-372, 2001.

20. Laing NG, Dye DE, Wallgren-Pettersson C, Richard G, Monnier N, Lillis S, Winder TL, Lochmuller H, Graziano C, MitraniRosenbaum S, Twomey D, Sparrow JC, Beggs AH and Nowak KJ: Mutations and polymorphisms of the skeletal muscle alpha-actin gene (ACTA1). Hum Mutat 30: 1267-1277, 2009.

21. Smith CK, 2nd, Janney MJ and Allen RE: Temporal expression of myogenic regulatory genes during activation, proliferation, and differentiation of rat skeletal muscle satellite cells. J Cell Physiol 159: 379-385, 1994. 